\title{
BIOCHEMICAL CHANGES AND MANIFESTATIONS OF ENVENOMATION PRODUCED BY Odonthobuthus doriae VENOM IN RABBITS
}

MIRAKABADI A. Z. (1), JALALI A. (2), JAHROMI A. E. (3), VATANPUR H. (3),

\section{AKBARY A. (1)}

1) Razi Vaccine and Serum Research Institute, Hessarak Karaj, Iran; (2) Department of Toxicology and Pharmacology, School of Pharmacy, Jundishapour University of Medical Sciences, Ahwaz, Iran, (3) Department of Toxicology and Pharmacology, School of Pharmacy, Shaheed Beheshti University of Medical Sciences, Tehran, Iran.

ABSTRACT: Many toxins from scorpion venoms cause neurotransmitters release by activating the autonomic system. The aim of the present work was to determine osmotic fragility of red blood cells (RBCs) and serum biochemical changes produced by the venom of Odonthobuthus doriae (O. doriae), a dangerous species of scorpion in Iran. For this study we selected 2 groups, each one containing $10 \mathrm{New}$ Zealand white rabbits weighing $2 \pm 0.2 \mathrm{~kg}$. In vivo and in vitro osmotic fragilities as well as packed cell volume (PCV) were determined. Serum was separated and used for determination of glucose, blood urea nitrogen (BUN), creatinine, uric acid (UA), triglycerides, cholesterol, aspartate aminotransferase (AST, EC 2.6.1.1), and alanine aminotransferase (ALT, EC 2.6.1.2). Results indicate that Odonthobuthus doriae venom $(0.5 \mathrm{mg} / \mathrm{kg}$, IV) causes a significant increase $(p<0.05)$ of serum glucose, UA, PCV, ALT, and AST. Increase was also observed in BUN, but it was not statistically significant. On the other hand a significant decrease $(p<0.05)$ was observed in triglycerides and cholesterol levels. Increased in vivo osmotic fragility of RBCs was significant too, but in vitro osmotic fragility did not show a significant change. These results support the hypothesis that the biochemical variation caused by scorpion venom can be due to an autonomic storm and release of catecholamines.

KEY WORDS: scorpion venom, Odonthobuthus doriae, biochemical changes, manifestation of envenomation, osmotic fragility of RBCs.

\section{CORRESPONDENCE TO:}

ABBAS ZARE MIRAKABADI, Department of Biochemistry, Razi Vaccine and Serum Research Institute, Hesarak, Karaj, Iran. Phone: 0098261 4570038. Email: zare@zare.ir 


\section{INTRODUCTION}

Most scorpion venom effects are attributable to the ability of their constituents to act on the somatic or autonomic nervous systems, which facilitates catecholamines release. Stimulatory effects of scorpion venom on neurotransmitters release, including acetylcholine (6) and noradrenalin (16), have been shown by various researchers. Later, it was demonstrated that scorpion envenomation causes an autonomic storm releasing massive amounts of catecholamines $(15,20)$.

Hypotheses that believed manifestations of scorpion envenomation were due to the enhanced release of neurotransmitters were further supported by the Mesobuthus tamulus venom action $(17,27)$. Studies have shown that Mesobuthus tamulus venom causes pancreatitis with an increase in the exocrine secretion of amylase (18). Azevedo et al. show that cardiovascular and respiratory effects of Tityus serrulatus venom are a consequence of acetylcholine release (3). Androctonus crassicauda is the only scorpion in Iran whose clinical effects were studied (25). In preliminary studies, our group observed that $O$. doriae venom causes positive inotropic arrhythmic effects and increased contractility of the cardiac muscle in guinea pigs. Since pretreatment of heart with propanolol blocks inotropic and increases contractility effects of $O$. doriae venom, we can infer this venom may have an effect on the autonomic system and enhance the release of catecholamines similarly to some other Buthidae family scorpion venoms.

In an attempt to assess the role of $O$. doriae venom-induced release of catecholamines and its effects on the nervous system, this study was designed to investigate in vivo effects of the venom on biochemical parameters and the osmotic fragility of RBCs, in vivo and in vitro, in experimental rabbits.

\section{MATERIALS AND METHODS}

\section{Material}

Crude scorpion venom was donated by Dr. Akbary, head of the Department of Venomous Animals, Razi Vaccine and Serum Research Institute, Karaj, Iran. It was collected by electrically stimulating the telson of scorpion and was freeze-dried. The venom was reconstituted in $0.9 \%$ sodium chloride solution to a proper concentration. Except for heparin sodium, which was prepared from Leo® (Denmark), and definite kits purchased from Sigma ${ }^{\circledR}$, all other materials were supplied from either Merck® or Fluka®. 
Two groups of New Zealand white rabbits weighing $2 \pm 0.2 \mathrm{~kg}$ (10 animals in each group), one group as control and the other one as experimental, were selected and kept under similar feeding conditions for study.

\section{Methods}

Saline and venom $(0.5 \mathrm{mg} / \mathrm{kg}$, IV) injection, and blood sampling were carried out via a polyethylene canola (G21) inserted into the marginal ear vein. Blood sampling was performed after overnight fasting, before and one hour after venom and saline administration. Experiments ended with the death of rabbits (100 min average) after venom injection; surviving animals, as control, were killed 2 hours after salinetreatment by overdose of diethyl ether. Post-mortem examinations of lungs were carried out and the lung weight / body weight x 100 index was calculated (2).

Blood collected in tubes containing EDTA was processed for in vivo osmotic fragility (24) and hematocrit (5). Serum was processed for glucose, free fatty acids, triglycerides (24), AST, ALT and LDH (L-lactate dehydrogenase) - kits supplied from Sigma ${ }^{\circledR}$ - creatinine, BUN, uric acid, and cholesterol $(5,26)$.

Osmotic fragility of RBCs was tested after 30 minutes and 24 hours of incubation in vivo. Crude venom at the concentrations of $0,0.03,0.06,0.09$, and $0.1 \mathrm{mg} / \mathrm{ml}$ blood was incubated in vitro and processed for osmotic fragility $(21,24)$.

\section{Statistical analysis}

The biochemical parameters measurements were expressed as the mean \pm standard error of mean (SEM). Osmotic fragility values were expressed as the mean of 10 rabbits. Statistical significance in cases of repeated measurements within group, before and after venom / saline treatments, was evaluated by the student T-test. Values of $p<0.05$ were considered significant. All the parameters were determined for 10 rabbits, except LDH, which was carried out for 3 rabbits.

\section{RESULTS}

\section{Manifestations of scorpion envenomation}

Signs of envenomation were studied 15-25 min after venom injection. Salivation, lacrimation, and muscle excitement along with bronchial secretion and labored breathing indicating pulmonary edema in the later stages were seen in all the animals. In some animals, respiratory changes, comprising periods of bradypnea 
alternating with tachypnea, were also seen. Agitation and tremor of the face was occasionally observed in the animals. Post-mortem examination of lungs revealed the average lung weight / body weight index for venom-treated rabbits $(0.43 \pm 0.02)$ was significantly higher than that of control, saline-treated rabbits $(0.3 \pm 0.03$, $p<0.05)$. Death occurred in an average of 100 min after injection.

\section{Metabolic changes}

Blood glucose levels, AST, and ALT were increased after venom injection, 152.2\%, $126 \%$, and $111.2 \%$, respectively, which were statistically significant. Triglycerides levels decreased significantly $(p<0.005)$ to $31.1 \%$. However, we obtained results showing decreased LDH activity, but this change was not significant (Table 1).

Creatinine and BUN levels increased, and cholesterol presented a decreased level. However these changes were not statistically significant. A significant increase was observed in hematocrit $(p<0.05)$ and uric acid $(p<0.005)$, respectively (Table 2$)$.

\section{Osmotic fragility of erythrocytes in vivo and in vitro}

Increased osmotic fragility of RBCs was highly significant $(p<0.005)$ and significant $(p<0.05)$ after $30 \mathrm{~min}$ and 24 hours incubation in vivo, respectively. Lyses of RBCs with different sodium chloride concentrations occurred at $0.55 \%$ and $0.8 \%$ after 30 min and 24 hours incubation, respectively (Figures 1 and 2). Data were compared with maximum fragility (100\%) at $0.1 \%$ sodium chloride concentration.

Significant changes in osmotic fragility of RBCs were not observed after 30 min and 24 hours incubation in vitro at the venom concentrations $0.03,0.06$, and $0.1 \mathrm{mg} / 2$ $\mathrm{ml}$ of blood (Figures 3 and 4). Data were compared with maximum fragility $(100 \%)$ at $0.1 \%$ sodium chloride concentration. 
Table 1: Effects of $O$. doriae venom on rabbits serum ALT, AST, LDH, glucose, and triglycerides (mean \pm SEM).

NS: Not significant.

\begin{tabular}{l|l|l|l|l}
\hline Parameters & Before venom & After venom* & \% changes & $p$ value \\
\hline ALT $(\mathrm{U} / \mathrm{l})$ & $17.8 \pm 2.3$ & $37.8 \pm 3.9$ & +111.2 & $p<0.005$ \\
\hline AST $(\mathrm{U} / \mathrm{l})$ & $12.9 \pm 2.3$ & $29.2 \pm 4.5$ & +126 & $p<0.001$ \\
\hline LDH $(\mathrm{U} / \mathrm{l})$ & $485 \pm 43.1$ & $370 \pm 39.2$ & -23.7 & $\mathrm{NS}$ \\
\hline Glucose $(\mathrm{mg} / \mathrm{dl})$ & $153.7 \pm 7.5$ & $310.8 \pm 39.8$ & +102.2 & $p<0.005$ \\
\hline Triglycerides $(\mathrm{mg} / \mathrm{dl})$ & $130 \pm 10.4$ & $89 \pm 39.8$ & -31.1 & $p<0.05$ \\
\hline
\end{tabular}

$\%$ changes: Changes between before and after venom administration.

*: $0.5 \mathrm{mg} / \mathrm{kg}, \mathrm{IV}$.

Table 2: Effects of $O$. doriae venom on rabbits serum uric acid, BUN, creatinine, hematocrit, and cholesterol (mean \pm SEM).

\begin{tabular}{l|l|l|l|l}
\hline Parameters & Before venom & After venom* & \% changes & $p$ value \\
\hline BUN $(\mathrm{mg} / \mathrm{dl})$ & $30.20 \pm 3$ & $38.20 \pm 3.9$ & +26 & NS \\
\hline Creatinine $(\mathrm{mg} / \mathrm{dl})$ & $0.837 \pm 0.12$ & $1.87 \pm 0.3$ & +123 & NS \\
\hline Uric acid $(\mathrm{mg} / \mathrm{dl})$ & $1.20 \pm 0.2$ & $3.80 \pm 0.59$ & +217 & $p<0.005$ \\
\hline Hematocrit & $37.70 \pm 0.7$ & $43.5 \pm 0.8$ & +15 & $p<0.05$ \\
\hline Cholesterol $(\mathrm{mg} / \mathrm{dl})$ & $77.00 \pm 2.8$ & $66.0 \pm 4.5$ & -14.3 & NS \\
\hline
\end{tabular}

NS: Not significant.

$\%$ changes: Changes between before and after venom administration.

*: $0.5 \mathrm{mg} / \mathrm{kg}$, IV. 


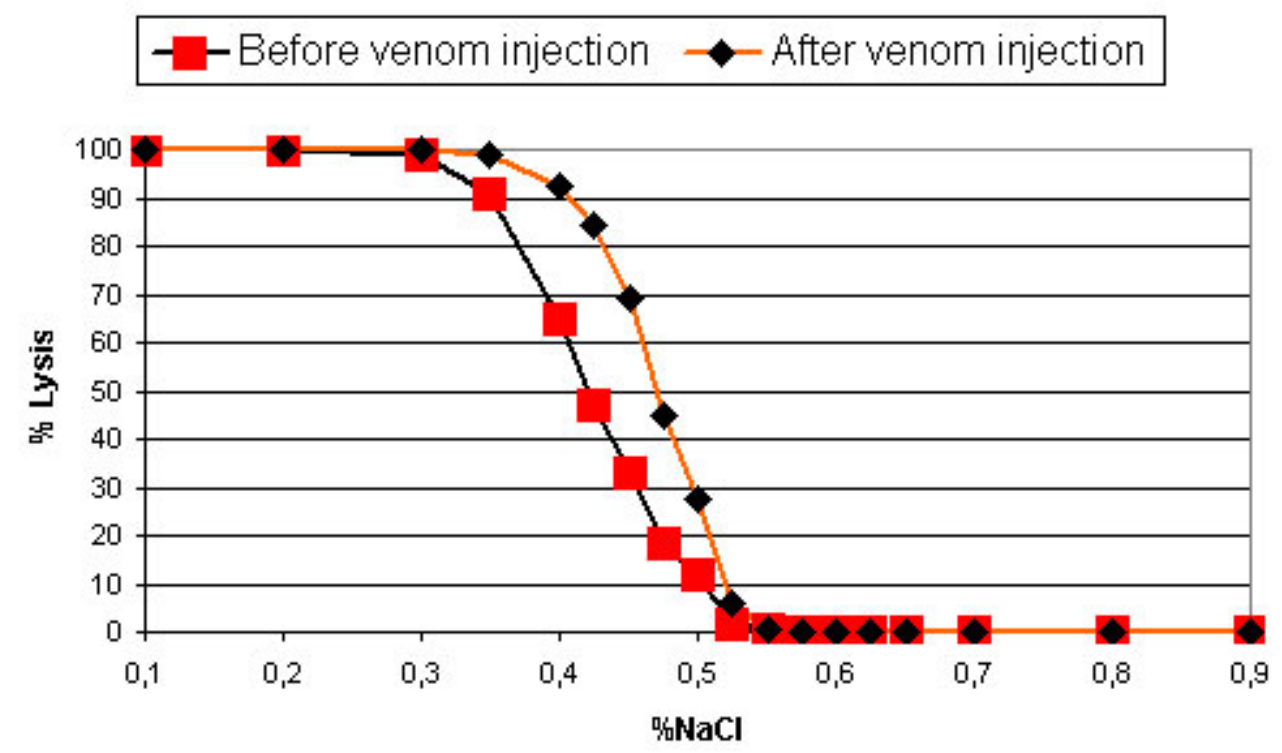

Figure 1: In vivo effect of $O$. doriae venom $(0.5 \mathrm{mg} / \mathrm{kg}$, IV) on the osmotic fragility of RBCs after incubation for 30 minutes.

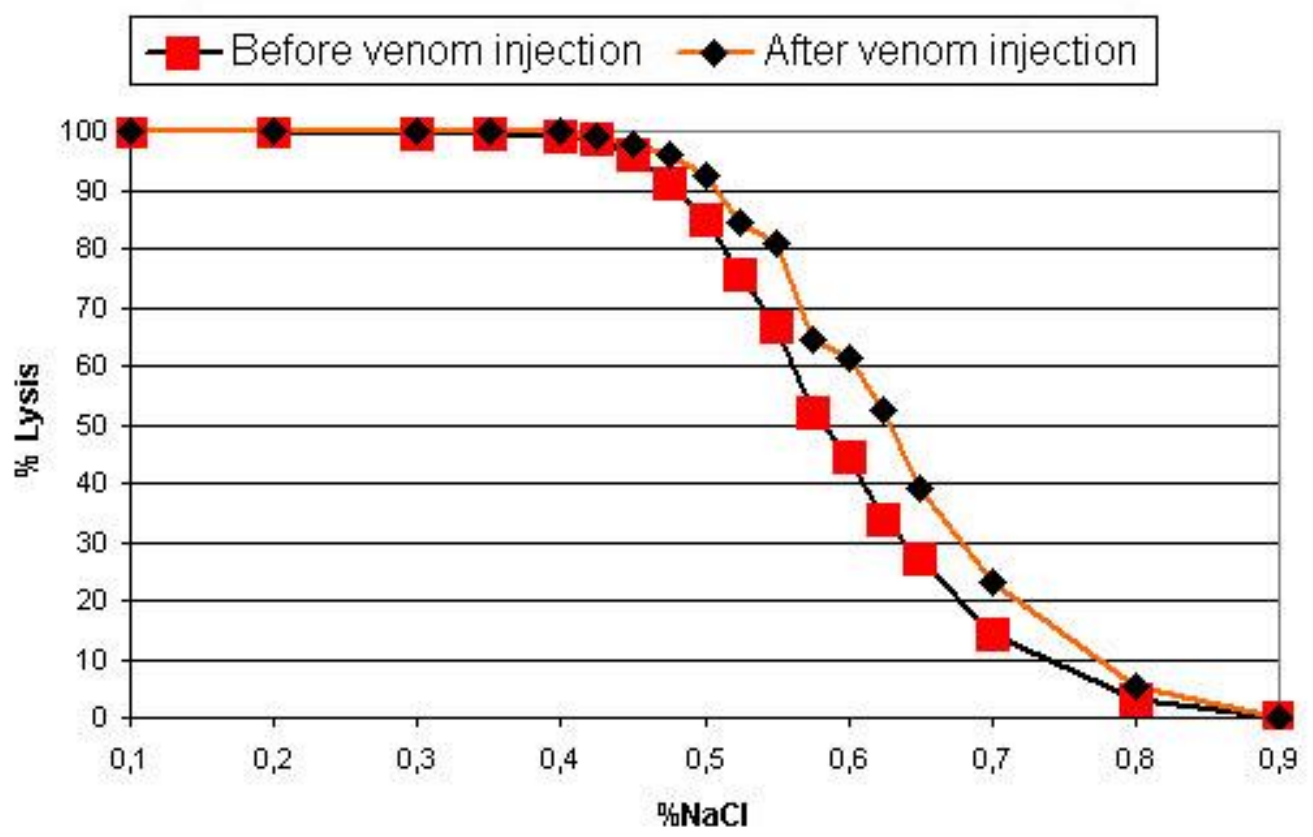

Figure 2: In vivo effect of $O$. doriae venom $(0.5 \mathrm{mg} / \mathrm{kg}$, IV) on the osmotic fragility of RBCs after incubation for 24 hours. 


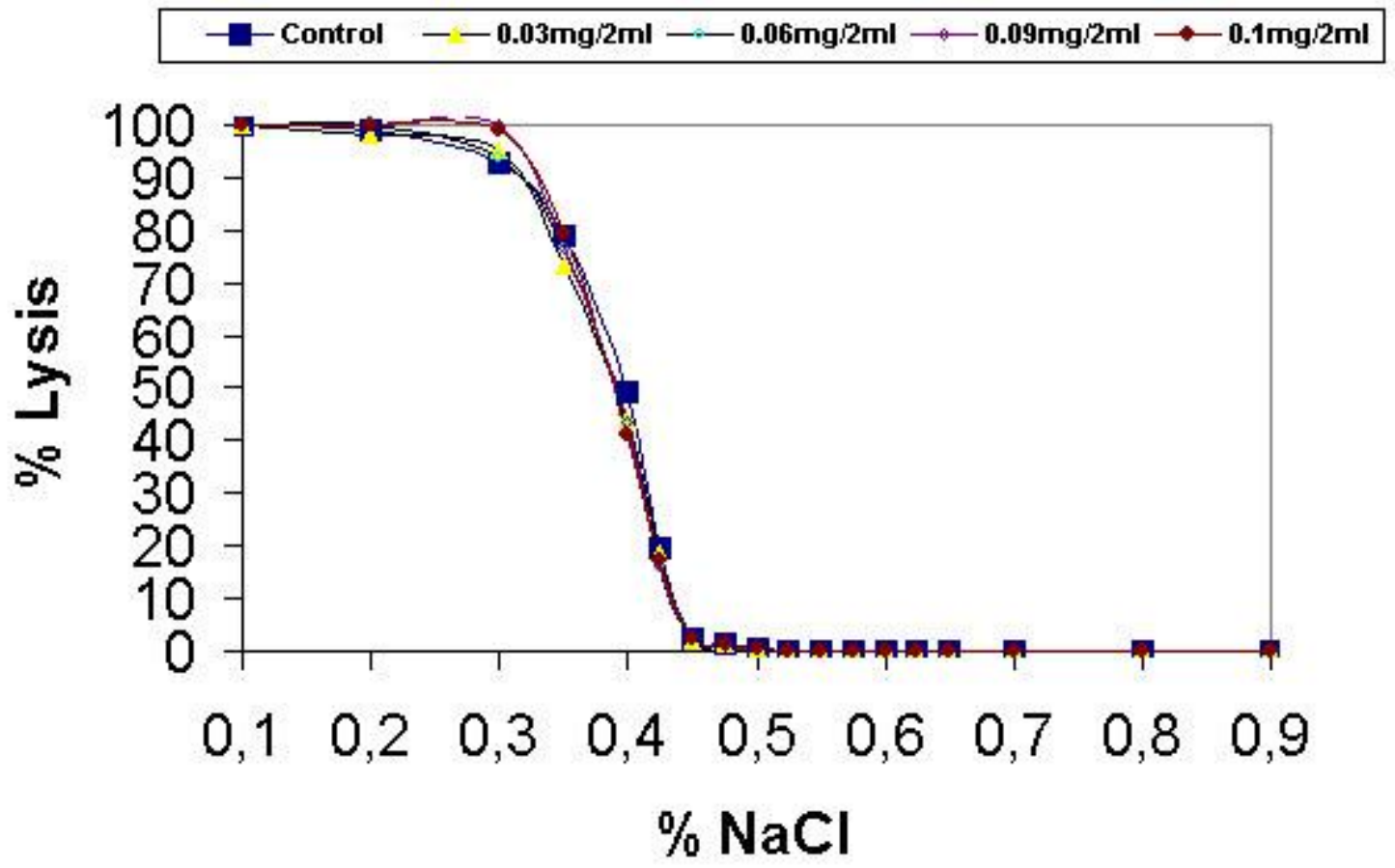

Figure 3: In vitro effect of $O$. doriae venom $(0.03,0.06,0.09$, and $0.1 \mathrm{mg} / 2 \mathrm{ml}$ of blood) on the osmotic fragility of RBCs after incubation for 30 minutes.

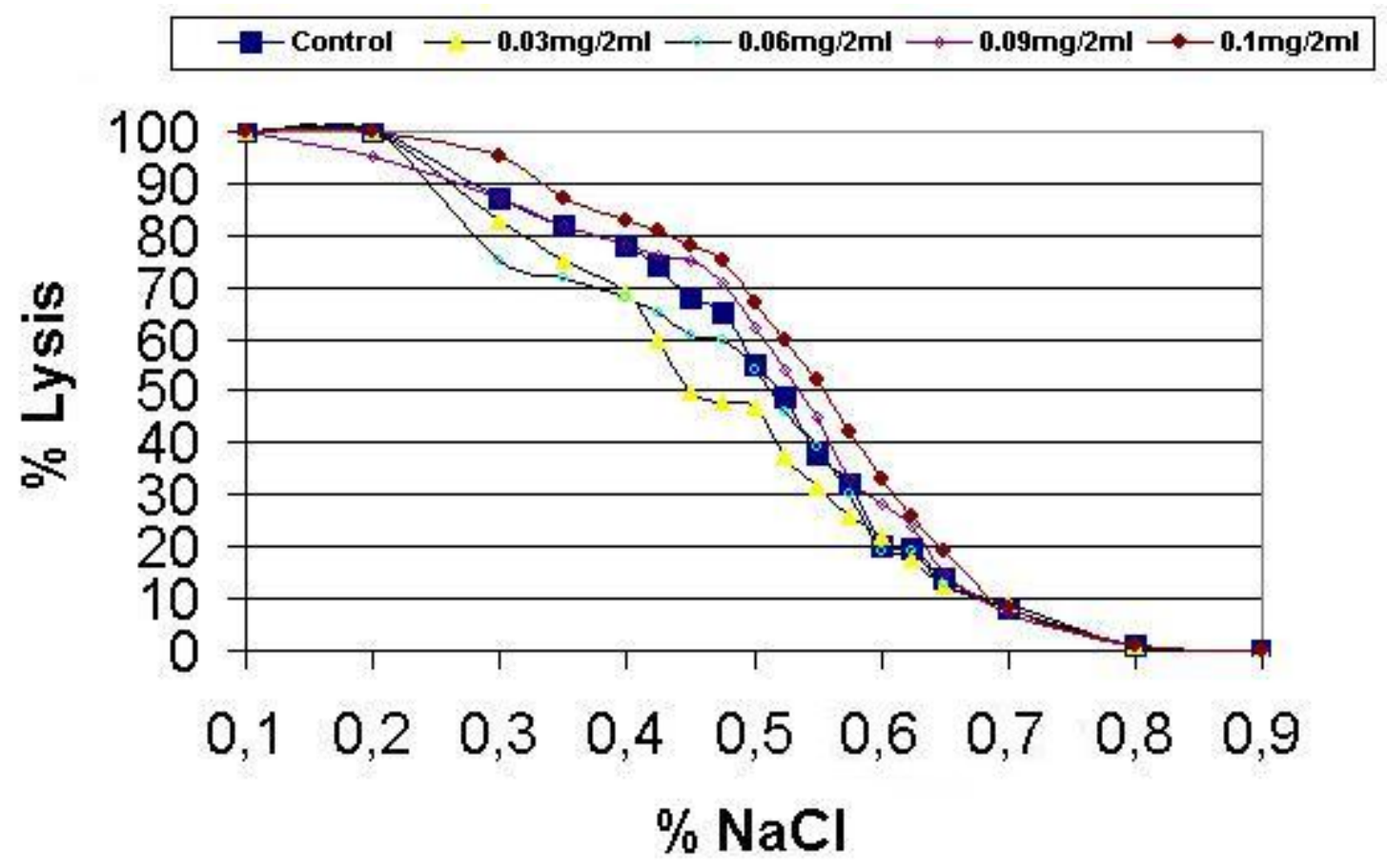

Figure 4: In vitro effect of $O$. doriae venom $(0.03,0.06,0.09$, and $0.1 \mathrm{mg} / 2 \mathrm{ml}$ of blood) on the osmotic fragility of RBCs after incubation for 24 hours. 


\section{DISCUSSION}

Clinical manifestations of scorpion envenomation appear to be secondary to the activation of both sympathetic and parasympathetic autonomic nervous system. This manifestation confirms that scorpion envenomation causes an autonomic storm and this fact was also present in other researches $(10,11,15,17)$. In the present study, significant hyperglycemia was seen after $O$. doriae venom administration. Our findings in hyperglycemia measurement are quite compatible to the results from other studies $(1,8,13,17,23,27)$. The proposed mechanism for this effect included: peripheral and central stimulation of the adrenergic system $(8,13,27)$, activation of $\beta$-receptors with catecholamine and serotonin secretion (1), block of insulin secretion (8), and insulin resistance (27). However, the results of Zare and Ismail demonstrated contraries in relation to hyperglycemia along with hyperinsulinemia $(13,27)$. This could be due to differences in the dose and route of administration. We believe that hyperinsulinemia is due to insulin resistance rather than hyperglycemia after scorpion venom injection.

The level of triglycerides decreased after venom injection. Some reports notify that the free fatty acids level increases significantly after scorpion venom administration. Our results also confirm previous reports $(7,19,27)$. Stimulatory effect of catecholamines on the breaking down of triglycerides has been confirmed in vivo and in vitro (9).

Cholesterol level reduced in this study as previously demonstrated with another scorpion venom $(13,23)$. This effect could be due to hepatic tissue destruction and hence increased cholesterol excretion through bile or to hepatic failure in biosynthesis of cholesterol.

Increase of AST and ALT levels in the envenomed animals is in agreement with former reports $(23,27)$. AST as a transferase enzyme is distributed in all parts of the body, but mostly concentrates in the liver and heart, therefore the increase of AST level is attributed to myocardial infarction or hepatic failure. ALT, with similar activity to AST, is considered as a liver-specific enzyme. It increases more and remains longer than AST in hepatic failure or inflammation (22). Therefore, the increase of AST and ALT levels may be due to a direct action of the venom on liver and heart. Increases in BUN, uric acid, and creatinine in this work are in accordance with other studies $(13,23)$. They are the final products of protein metabolism, and their 
concentration would increase in renal failure. Renal failure and decrease of glomerular filtration may be related to cardiac failure (23).

Hematocrit or packed cell volume (PCV) was increased. This finding is in accordance with previous reports on Mesobuthus tamulus venom (27), but it is contrary to the findings of Ismail in 1988 (13). This effect may be due to an increase in angiotensin II which has been further emphasized by Murthy $(14,22)$.

Increased osmotic fragility of erythrocytes in vivo is supported by other studies (14, 27). This could be due to hyperthermia and acidosis following scorpion envenomation $(4,12,27)$. However increased osmotic fragility after 24 hours is attributed to the increase of free fatty acids and block of $\mathrm{Na}^{+}-\mathrm{K}^{+}$-ATPase (4). No change in the osmotic fragility in vitro indicates that there is no direct action of $O$. doriae venom on RBCs.

Hence, according to the results obtained, this study supports the hypothesis that $O$. doriae venom, like other dangerous scorpion venoms, enhances release of catecholamines with consequent stimulation of the somatic and autonomic nervous system. In addition, this study suggests that $O$. doria venom may be inefficient in the utilization of metabolic substances by its direct action on the heart and/or liver, inhibition of insulin secretion, and insulin resistance, but further studies are needed to provide more evidences for validating this hypothesis.

\section{REFERENCES}

1 ADAM KR., WEISS C. Distribution of 5-hydroxytryptamine in scorpion venoms. Nature, 1959, 183, 1389-99.

2 ALMEIDA HO., LIMA EG., FREIRE-MAIA L. Mechanism of the acute pulmonary edema induced by intracerebroventricular injection of scorpion toxin (tityustoxin) in the unanesthetized rat. Toxicon, 1976, 14, 435-40.

3 AZEVEDO AD., SILVA AB., CUNHA-MELO JR., FREIRE-MAIA L. Cardiovascular and respiratory effects induced by a purified scorpion (Tityustoxin) in the unanesthetized rats. Toxicon , 1983, 21, 753-9.

4 BALASUBRAMANIAM P., MURTHY KR. Liver glycogene depletion in acute myocarditis produced by scorpion venom (Buthus tamulus). Indian Heart J., 1984, 36, 101-3.

5 DACIE JV., LEWIS SM. Practical Haematology. 6.ed. Edinburgh: Churchill Livingstone, 1984, 453p. 
6 DINIZ CR., TORRES JM. Release of an acetylcholine-like substance from guinea pig ileum by scorpion venom. Toxicon, 1968, 5, 277-81.

7 EL-ASMAR MF., FARAG RM., SHOUKRY S., EL-SMIMI IR. Effect of scorpion (Leiurus quinquestriatus $\mathrm{H}$ and $\mathrm{E}$ ) venom on lipid metabolism. Toxicon, 1979, 17, 279-83.

8 EL-ASMAR MF., SOLIMAN SF., ISMAIL M., OSMAN OH. Glycemic effect of venom from the scorpion Buthus minax (L.Koch). Toxicon, 1974, 12, 249-51.

9 GUERON M., OVSYSHCHER I. What is the treatment for the cardiovascular manifestations of scorpion envenomation? Toxicon, 1987, 25, 121-30.

10 ISMAIL M. The treatment of the scorpion envenoming syndrome: the Saudi experience with serotherapy. Toxicon, 1994, 32, 1019-26.

11 ISMAIL M. The scorpion envenoming syndrome. Toxicon, 1995, 33, 825-58.

12 ISMAIL M., ABD-ELSALAM MA. Are the toxicological effects of scorpion envenomation related to tissue venom concentration? Toxicon, 1988, 26, 233-56.

13 ISMAIL M., ABD-ELSALAM MA., MORAD AM. Do changes in body temperature following envenomation by the scorpion Leiurus quinquestriatus influence the course of toxicity? Toxicon, 1990, 28, 1265-84.

14 KARI RK. ZOLFAGHRIAN H. Increased osmotic fragility of red cells in dogs with acute myocarditis produced by scorpion (Buthus tamulus) venom. Indian J. Physiol. Pharmacol., 1986, 30, 215-22.

15 MAZZEI DE DAVILA CA., DAVILA DF., DONIS JH,.BELLABARBA GA., VILLARREAL V., BARBOZA JS. Sympathetic nervous system activation, antivenin administration and cardiovascular manifestation of scorpion envenomation. Toxicon, 2002, 40, 1339-46.

16 MOSS J., THOA NB., KOPIN IJ. On the mechanism of scorpion toxin-induced release of norepinephrine from peripheral adrenergic neurons. J. Pharmacol. Exp. Ther., 1974, 190, 39-48.

17 MURTHY KR., BILLIMORIA FR., KHOPKAR M., DAVE KN. Acute hyperglycemia and hyperkalaemia in acute myocarditis produced by scorpion (Buthus tamulus) venom injection in dogs. Indian Heart J., 1986, 38, 71-4. 
18 MURTHY KR., MEDH JD., DAVE BN., VAKIL YE., BILLIMORIA FR., Acute pancreatitis and reduction of $\mathrm{H}^{+}$ion concentration in gastric secretions in experimental acute myocarditis produced by Indian red scorpion (Buthus tamulus) venom. Indian J. Exp. Biol., 1989, 27, 242-4.

19 MURTHY KR., VAKIL AE. Elevation of plasma angiotensin levels in dogs by Indian red scorpion (Buthus tamulus) venom and its reversal by administration of insulin + tolazoline. Indian J. Med. Res., 1988, 88, 376-9.

20 MURTHY KR., ZARE MA. Effect of Indian red scorpion (Mesobuthus tamulus concanesis, Pocock) venom on thyroxine and triiodothyronine in experimental acute myocarditis and its reversal by specific antivenom. Indian J. Exp. Biol, 1998, 36, 16-21.

21 MURTHY KR., ZOLFAGHARIAN H., MEDH JD., KUDALKAR JA., YEOLEKAR ME., PANDIT SE., KHOBKAR M., DAVE KN., BILLIMORIA FR. Disseminated intravascular coagulation and disturbances in carbohydrate and fat metabolism in acute myocarditis produced by scorpion (Buthus tamulus) venom. Indian J. Med. Res., 1988, 87, 318-25.

22 MURTHY RR., VAKIL AE., YEOLEKAR KE. Insulin administration reverses the metabolic and electrocardiographic changes in acute myocarditis induced by Indian red scorpion (Buthus tamulus) venom in experimental dogs. Indian Heart J., 1990, 42, 35-42.

23 OMRAN MA., ABDEL-RAHMAN MS. Effect of scorpion Leiurus quinquestriatus ( $H$ and $E$ ) venom on the clinical chemistry parameters of the rat. Toxicol. Lett., 1992, 61, 99-109.

24 PARPART AK., LORENZ PB., PARAPART ER., GREGG JR., CHASE AM. The osmotic resistance (fragility) of human red cells. J. Clin. Invest., 1947, 26, 63640.

25 RADMANESH M. Study of scorpion sting in Khuzestan, Iran. Daru va Darman, 1986, 3, 12-9.

26 VARLEY H. Practical Clinical Biochemistry. 5.ed. London: Heinemann Medical, 1980. $1277 p$.

27 ZARE MA. Scorpion venom poisoning: reversal of biochemical hormonal and pathophysiological disturbances by antivenom and insulin therapy. Bombay, India: University of Bombay, 1993. 229 p. [Dissertation - Doctorate]. 